\title{
A BERNSTEIN THEOREM FOR MINIMAL MAPS WITH SMALL SECOND FUNDAMENTAL FORM
}

\author{
FELIX LUBBE
}

\begin{abstract}
We consider minimal maps $f: M \rightarrow N$ between Riemannian manifolds $\left(M, \mathrm{~g}_{M}\right)$ and $\left(N, \mathrm{~g}_{N}\right)$, where $M$ is compact and where the sectional curvatures satisfy $\sec _{N} \leq \sigma \leq \sec _{M}$ for some $\sigma>0$. Under certain assumptions on the differential of the map and the second fundamental form of the graph $\Gamma(f)$ of $f$, we show that $f$ is either the constant map or a totally geodesic isometric immersion.
\end{abstract}

\section{Contents}

1. Introduction 1

2. The Strong Elliptic Maximum Principle 3

3. Maps between Manifolds 4

4. Bernstein-type Theorems 6

5. Discussion 13

$\begin{array}{ll}\text { References } & 14\end{array}$

\section{Introduction}

We consider smooth minimal maps $f: M \rightarrow N$ between Riemannian manifolds $\left(M, \mathrm{~g}_{M}\right)$ and $\left(N, \mathrm{~g}_{N}\right)$. The map $f$ is called minimal, if its graph

$$
\Gamma(f):=\{(x, f(x)): x \in M\} \quad \subset M \times N
$$

is a minimal submanifold of $\left(M \times N, \mathrm{~g}_{M} \times \mathrm{g}_{N}\right)$ [13]. The Bernstein theorem asserts that any complete minimal surface in $\mathbb{R}^{3}$ which can be written as a graph of a function on $\mathbb{R}^{2}$ must be a plane. This result has been generalized to $\mathbb{R}^{n}$ for $n \leq 7$ and to general dimensions under various growth conditions (see e.g. [2] and references therein).

A generalized Bernstein problem is to ask under which additional geometric conditions the graph $\Gamma(f)$ is totally geodesic. Several results for higher codimension have been obtained, e.g. by assuming conditions on the slope of the graph and by considering its Gauss map $[3,6,7,9,10,14,16]$.

Department of Mathematics, University of Hamburg, Bundesstr. 55, D-20146 HamBurg, Germany

E-mail address: Felix.Lubbe@uni-hamburg.de.

2010 Mathematics Subject Classification. Primary 53C42; 53C40, 58J05.

Key words and phrases. Minimal maps, Bernstein theorem. 
Let us assume that $M$ is compact. A further approach to the problem is to demand a volume-decreasing condition for the map $f$. The map $f$ is called weakly lengthdecreasing if

$$
\|\mathrm{d} f(v)\|_{\mathrm{g}_{N}} \leq\|v\|_{\mathrm{g}_{M}} \quad \text { for all } \quad v \in \Gamma(T M)
$$

and weakly area-decreasing if

$$
\|\mathrm{d} f(v) \wedge \mathrm{d} f(w)\|_{\mathrm{g}_{N}} \leq\|v \wedge w\|_{\mathrm{g}_{M}} \quad \text { for all } \quad v, w \in \Gamma(T M) .
$$

The length-decreasing property may be expressed in terms of the symmetric tensor

$$
\mathrm{s}:=\mathrm{g}_{M}-f^{*} \mathrm{~g}_{N},
$$

i. e. $f$ is weakly length-decreasing precisely if $\mathrm{s}$ is non-negative definite. This tensor satisfies a nice differential equation, and allows the application of a tensorial maximum principle (see [12], also for the area-decreasing case).

Instead of considering the above tensor and its eigenvalues directly, we will study the behavior of minimal maps in relation to the trace

$$
\operatorname{tr}(\mathrm{s}):=\sum_{k=1}^{m} \mathrm{~s}\left(e_{k}, e_{k}\right),
$$

where $\left\{e_{1}, \ldots, e_{m}\right\}$ is a local frame of $T M$, orthonormal with respect to the metric $\mathrm{g}$ induced by the embedding $\Gamma(f) \subset M \times N$. We remark that $\frac{1}{m} \operatorname{tr}(\mathrm{s})$ is the mean value of the eigenvalues of s, i. e. the trace also may be seen as an average. After these preparations, we are able to state the main result.

Theorem A. Let $\left(M, \mathrm{~g}_{M}\right)$ and $\left(N, \mathrm{~g}_{N}\right)$ be Riemannian manifolds and suppose $M$ to be compact of dimension $\operatorname{dim} M \geq 2$. Assume that there exists $\sigma>0$, such that the sectional curvatures $\sec _{M}$ of $M$ and $\sec _{N}$ of $N$ satisfy the relation

$$
\sec _{N} \leq \sigma \leq \sec _{M}
$$

Let $f: M \rightarrow N$ be a minimal map and assume that the tensor s satisfies the condition

$$
\operatorname{tr}(\mathrm{s}) \geq 0 \text {. }
$$

Further, assume there is a finite constant $\kappa$, such that

$$
\kappa^{2}>1 \quad \text { and } \quad f^{*} \mathrm{~g}_{N}<\kappa^{2} \mathrm{~g}_{M}
$$

and such that the second fundamental tensor A of the graph satisfies the bound

$$
\|\mathrm{A}\|^{2} \leq \frac{\kappa^{2} \sigma}{\kappa^{4}-1} \operatorname{tr}(\mathrm{s}) .
$$

Then one of the following holds.

(i) $f$ is the constant map.

(ii) $f$ is a totally geodesic isometric immersion, $\sec _{M}=\sigma$ and the restriction of $\sec _{N}$ to $\mathrm{d} f(T M)$ is equal to $\sigma$. Moreover, $\Gamma(f)$ is a totally geodesic submanifold of $M \times N$.

If the target space has at most half the dimension of the domain, the condition on the trace is automatically satisfied, i. e. the following statement holds.

Corollary B. Let $\left(M, \mathrm{~g}_{M}\right)$ be compact of dimension $m=\operatorname{dim} M \geq 2$, let $\left(N, \mathrm{~g}_{N}\right)$ be of dimension at most $\frac{m}{2}$, and assume $\sec _{N} \leq \sigma \leq \sec _{M}$ for some $\sigma>0$. If a minimal map $f: M \rightarrow N$ satisfies the condition (1.2), then $f$ is the constant map. 
Let us shortly describe the structure of the paper. In section 2, we recall the strong elliptic maximum principle in vector bundles [12], as required by the subsequent sections. Section 3 describes the geometry of graphs, and section 4 provides the proof of theorem A. The paper ends with a short discussion of the assumptions in section 5.

Acknowledgements. I am grateful to Andreas Savas-Halilaj for valuable discussions. This research was initiated while I was supported by the Research Training Group 1463 of the DFG at the Leibniz Universität Hannover.

\section{The Strong Elliptic Maximum Principle}

In [12], Savas-Halilaj and Smoczyk provided a strong elliptic maximum principle for vector bundles, which we would like to apply. We briefly collect the results relevant for us. All manifolds will be smooth and connected without boundary.

Let $(E, \pi, M)$ be a vector bundle of rank $k$ over a smooth manifold $M$. Suppose $g_{E}$ is a bundle metric on $E$ and that $\nabla$ is a metric connection on $E$. A uniformly elliptic operator $\mathscr{L}$ on $\Gamma(E)$ of second order is locally given by

$$
\mathscr{L}=\sum_{i, j=1}^{m} a^{i j} \nabla_{e_{i}, e_{j}}^{2}+\sum_{j=1}^{m} b^{j} \nabla_{e_{j}},
$$

where $a \in \Gamma(T M \otimes T M)$ is a symmetric, uniformly positive definite tensor and $b \in \Gamma(T M)$ is a smooth vector field, such that

$$
a=\sum_{i, j=1}^{m} a^{i j} e_{i} \otimes e_{j} \quad \text { and } \quad b=\sum_{j=1}^{m} b^{j} e_{j}
$$

in a local frame field $\left\{e_{1}, \ldots, e_{m}\right\}$ of $T M$.

The following definition goes back to Hamilton [4, Sec. 9].

Definition 2.1. A fiberwise map $\Psi: \operatorname{Sym}\left(E^{*} \otimes E^{*}\right) \rightarrow \operatorname{Sym}\left(E^{*} \otimes E^{*}\right)$ is said to satisfy the null-eigenvector condition, if whenever $\vartheta$ is a non-negative symmetric 2-tensor at a point $x \in M$ and if $v \in T_{x} M$ is a null-eigenvector of $\vartheta$, then $\Psi(\vartheta)(v, v) \geq 0$.

The elliptic analogue of Hamilton's maximum principle [5, Lemma 8.2] is given by the next theorem.

Theorem 2.2 ([12, Theorem 2.3]). Let $\left(M, \mathrm{~g}_{M}\right)$ be a Riemannian manifold and let $(E, \pi, M)$ be a Riemannian vector bundle over the manifold $M$ equipped with a metric connection. Suppose that $\phi \in \operatorname{Sym}\left(E^{*} \otimes E^{*}\right)$ is non-negative definite and satisfies

$$
\mathscr{L} \phi+\Psi(\phi)=0,
$$

where $\Psi$ is a smooth fiberwise map satisfying the null-eigenvector condition. If there is an interior point of $M$ where $\phi$ has a null-eigenvalue, then $\phi$ must have a nulleigenvalue everywhere.

For $\phi \in \operatorname{Sym}\left(E^{*} \otimes E^{*}\right)$ a real number $\lambda$ is called eigenvalue of $\phi$ with respect to $\mathrm{g}_{E}$ at the point $x \in M$, if there exists a non-zero vector $v \in E_{x}:=\pi^{-1}(x)$, such that

$$
\phi(v, w)=\lambda \mathrm{g}_{E}(v, w),
$$

for any $w \in E_{x}$. Since the tensor $\phi$ is symmetric, it admits $k$ real eigenvalues $\lambda_{1}(x), \ldots, \lambda_{k}(x)$ at each point $x \in M$. We will always arrange the eigenvalues, such that

$$
\lambda_{1}(x) \leq \cdots \leq \lambda_{k}(x) .
$$


The following result is also due to Hamilton [4] (see also [12, Theorem 2.4]).

Theorem 2.3 (Second Derivative Criterion). Suppose that $\left(M, \mathrm{~g}_{M}\right)$ is a Riemannian manifold and $(E, \pi, M)$ a Riemannian vector bundle of rank $k$ over $M$ equipped with a metric connection $\nabla$. Let $\phi \in \operatorname{Sym}\left(E^{*} \otimes E^{*}\right)$ be a smooth symmetric 2-tensor. If the largest eigenvalue $\lambda_{k}$ of $\phi$ admits a local maximum $\lambda$ at an interior point $x_{0} \in M$, then

$$
(\nabla \phi)(v, v)=0 \quad \text { and } \quad(\mathscr{L} \phi)(v, v) \leq 0
$$

for all vector $v$ in the eigenspace

$$
\left\{v \in E_{x_{0}}: \phi(v, w)=\lambda g_{E}(v, w) \text { for all } w \in E_{x_{0}}\right\}
$$

of $\lambda$ at $x_{0}$ and for all uniformly elliptic second order operators $\mathscr{L}$.

\section{Maps between Manifolds}

3.1. Geometry of Graphs. We recall the geometric quantities in a graphical setting, where we follow the presentation in [12, Section 3.1].

Let $\left(M, \mathrm{~g}_{M}\right)$ and $\left(N, \mathrm{~g}_{N}\right)$ be Riemannian manifolds of dimensions $m$ and $n$, respectively. The induced metric on the product manifold will be denoted by

$$
\mathrm{g}_{M \times N}=\mathrm{g}_{M} \times \mathrm{g}_{N} \text {. }
$$

A smooth map $f: M \rightarrow N$ defines an embedding $F: M \rightarrow M \times N$, by

$$
F(x)=(x, f(x)), \quad x \in M .
$$

The graph of $f$ is defined to be the submanifolds $\Gamma(f):=F(M)$. Since $F$ is an embedding, it induces another Riemannian metric $\mathrm{g}:=F^{*} \mathrm{~g}_{M \times N}$ on $M$. The two natural projections

$$
\pi_{M}: M \times N \rightarrow M, \quad \pi_{N}: M \times N \rightarrow N,
$$

are submersions, that is they are smooth and have maximal rank. Note that the tangent bundle of the product manifold $M \times N$ splits as a direct sum

$$
T(M \times N)=T M \oplus T N .
$$

The four metrics $\mathrm{g}_{M}, \mathrm{~g}_{N}, \mathrm{~g}_{M \times N}$ and $\mathrm{g}$ are related by

$$
\begin{aligned}
\mathrm{g}_{M \times N} & =\pi_{M}^{*} \mathrm{~g}_{M}+\pi_{N}^{*} \mathrm{~g}_{N}, \\
\mathrm{~g} & =F^{*} \mathrm{~g}_{M \times N}=\mathrm{g}_{M}+f^{*} \mathrm{~g}_{N} .
\end{aligned}
$$

Now let us define the symmetric 2-tensors

$$
\begin{aligned}
\mathrm{s}_{M \times N} & :=\pi_{M}^{*} \mathrm{~g}_{M}-\pi_{N}^{*} \mathrm{~g}_{N}, \\
\mathrm{~s} & :=F^{*} \mathrm{~s}_{M \times N}=\mathrm{g}_{M}-f^{*} \mathrm{~g}_{N} .
\end{aligned}
$$

Note that $\mathrm{s}_{M \times N}$ is a semi-Riemannian metric of signature $(m, n)$ on the manifold $M \times N$.

The Levi-Civita connection $\nabla^{\mathrm{g}_{M \times N}}$ associated to the Riemannian metric $\mathrm{g}_{M \times N}$ on $M \times N$ is related to the Levi-Civita connections $\nabla^{\mathrm{g}_{M}}$ on $\left(M, \mathrm{~g}_{M}\right)$ and $\nabla^{\mathrm{g}_{N}}$ on $\left(N, \mathrm{~g}_{N}\right)$ by

$$
\nabla^{\mathrm{g}_{M \times N}}=\pi_{M}^{*} \nabla^{\mathrm{g}_{M}} \oplus \pi_{N}^{*} \nabla^{\mathrm{g}_{N}} .
$$

The corresponding curvature operator $\mathrm{R}_{M \times N}$ on $M \times N$ with respect to the metric $\mathrm{g}_{M \times N}$ is related to the curvature operators $\mathrm{R}_{M}$ on $\left(M, \mathrm{~g}_{M}\right)$ and $\mathrm{R}_{N}$ on $\left(N, \mathrm{~g}_{N}\right)$ by

$$
\mathrm{R}_{M \times N}=\pi_{M}^{*} \mathrm{R}_{M} \oplus \pi_{N}^{*} \mathrm{R}_{N} .
$$


We denote the Levi-Civita connection on $M$ with respect to the induced metric $\mathrm{g}=F^{*} \mathrm{~g}_{M \times N}$ by $\nabla$ and the corresponding curvature tensor by $\mathrm{R}$.

The second fundamental tensor A of the graph $\Gamma(f)$ is defined as

$$
\mathrm{A}(v, w):=(\widetilde{\nabla} \mathrm{d} F)(v, w)=\nabla_{\mathrm{d} F(v)}^{\mathrm{g} M \times N} \mathrm{~d} F(w)-\mathrm{d} F\left(\nabla_{v} w\right),
$$

where $v, w \in \Gamma(T M)$ and $\widetilde{\nabla}$ is the connection on $F^{*} T(M \times N) \otimes T^{*} M$ induced by the Levi-Civita connection. The trace of A with respect to the metric $\mathrm{g}$ is called the mean curvature vector field of $\Gamma(f)$ and it will be denoted by

$$
\vec{H}:=\operatorname{tr} \mathrm{A} .
$$

Note that $\vec{H}$ is a section in the normal bundle of the graph. If $\vec{H}$ vanishes identically, the graph is said to be minimal. A map $f: M \rightarrow N$ between Riemannian manifolds is called minimal, if its graph $\Gamma(f)$ is a minimal submanifold of the product space $\left(M \times N, \mathrm{~g}_{M \times N}\right)[13]$.

3.2. Singular Value Decomposition. We recall the singular value decomposition theorem (see [12, Section 3.2] and the references cited there).

Fix a point $x \in M$, and let

$$
\lambda_{1}^{2}(x) \leq \lambda_{2}^{2} \leq \ldots \leq \lambda_{m}^{2}(x)
$$

be the eigenvalues of $f^{*} \mathrm{~g}_{N}$ with respect to $\mathrm{g}_{M}$. The corresponding values $\lambda_{i} \geq 0$, $i \in\{1, \ldots, m\}$, are called the singular values of the differential $\mathrm{d} f$ of $f$ and give rise to continuous functions on $M$. Let

$$
r:=r(x):=\operatorname{rank} \mathrm{d} f(x) .
$$

Obviously, $r \leq \min \{m, n\}$ and $\lambda_{1}(x)=\cdots=\lambda_{m-r}(x)=0$. At the point $x$ consider an orthonormal basis $\left\{\alpha_{1}, \ldots, \alpha_{m-r} ; \alpha_{m-r+1}, \ldots, \alpha_{m}\right\}$ with respect to $\mathrm{g}_{M}$ which diagonalizes $f^{*} \mathrm{~g}_{N}$. Moreover, at $f(x)$ consider a basis $\left\{\beta_{1}, \ldots, \beta_{n-r} ; \beta_{n-r+1}, \ldots, \beta_{n}\right\}$ that is orthonormal with respect to $\mathrm{g}_{N}$, and such that

$$
\mathrm{d} f\left(\alpha_{i}\right)=\lambda_{i}(x) \beta_{n-m+i},
$$

for any $i \in\{m-r+1, \ldots, m\}$. The above procedure is called the singular value decomposition of the differential $\mathrm{d} f$.

We construct a special basis for the tangent and the normal space of the graph in terms of the singular values. The vectors

$$
\widetilde{e}_{i}:= \begin{cases}\alpha_{i}, & 1 \leq i \leq m-r, \\ \frac{1}{\sqrt{1+\lambda_{i}^{2}(x)}}\left(\alpha_{i} \oplus \lambda_{i}(x) \beta_{n-m+i}\right), & m-r+1 \leq i \leq m,\end{cases}
$$

form an orthonormal basis with respect to the metric $\mathrm{g}_{M \times N}$ of the tangent space $\mathrm{d} F\left(T_{x} M\right)$ of the graph $\Gamma(f)$ at $x$. It follows that with respect to the induced metric $\mathrm{g}$, the vectors

$$
e_{i}:=\frac{1}{\sqrt{1+\lambda_{i}^{2}}} \alpha_{i}
$$

form an orthonormal basis of $T_{x} M$. Moreover, the vectors

$$
\xi_{i}:= \begin{cases}\beta_{i}, & 1 \leq i \leq n-r, \\ \frac{1}{\sqrt{1+\lambda_{i+m-n}^{2}(x)}}\left(-\lambda_{i+m-n}(x) \alpha_{i+m-n} \oplus \beta_{i}\right), & n-r+1 \leq i \leq n,\end{cases}
$$


form an orthonormal basis with respect to $\mathrm{g}_{M \times N}$ of the normal space $\mathcal{N}_{x} M$ of the graph $\Gamma(f)$ at the point $x$. From the formulae above, we deduce that

$$
\mathrm{s}_{M \times N}\left(\widetilde{e}_{i}, \widetilde{e}_{j}\right)=\mathrm{s}\left(e_{i}, e_{j}\right)=\frac{1-\lambda_{i}^{2}(x)}{1+\lambda_{i}^{2}(x)} \delta_{i j}, \quad 1 \leq i, j \leq m .
$$

Therefore, the eigenvalues of the 2-tensor s with respect to $\mathrm{g}$ are given by

$$
\frac{1-\lambda_{1}^{2}(x)}{1+\lambda_{1}^{2}(x)} \geq \cdots \geq \frac{1-\lambda_{m-1}^{2}(x)}{1+\lambda_{m-1}^{2}(x)} \geq \frac{1-\lambda_{m}^{2}(x)}{1+\lambda_{m}^{2}(x)} .
$$

Moreover,

$$
\mathrm{s}_{M \times N}\left(\xi_{i}, \xi_{j}\right)= \begin{cases}-\delta_{i j}, & 1 \leq i \leq n-r, \\ -\frac{1-\lambda_{i+m-n}^{2}(x)}{1+\lambda_{i+m-n}^{2}(x)} \delta_{i j}, & n-r+1 \leq i \leq n,\end{cases}
$$

and

$$
\mathrm{s}_{M \times N}\left(\widetilde{e}_{m-r+i}, \xi_{n-r+j}\right)=-\frac{2 \lambda_{m-r+i}(x)}{1+\lambda_{m-r+i}^{2}(x)} \delta_{i j}, \quad 1 \leq i, j \leq r
$$

\section{Bernstein-type Theorems}

In order to show theorem A, we need to control the eigenvalues of the tensor s. For this, following the ideas of [12], let us define the tensor

$$
\Phi_{c}:=\mathrm{s}-\frac{1-c}{1+c} \mathrm{~g} .
$$

The differential equation satisfied by $\Phi_{c}$ was derived in [12, Lemma 3.2] and is given by

$$
\begin{aligned}
\left(\Delta \Phi_{c}\right)(v, w)=\mathrm{s}_{M \times N}\left(\nabla_{v} \vec{H}, \mathrm{~d} F(w)\right)+\mathrm{s}_{M \times N}\left(\nabla_{w} \vec{H}, \mathrm{~d} F(v)\right) \\
+2 \frac{1-c}{1+c} \mathrm{~g}_{M \times N}(\vec{H}, \mathrm{~A}(v, w)) \\
+\Phi_{c}(\operatorname{Ric} v, w)+\Phi_{c}(\operatorname{Ric} w, v) \\
+2 \sum_{k=1}^{m}\left(\mathrm{~s}_{M \times N}-\frac{1-c}{1+c} \mathrm{~g}_{M \times N}\right)\left(\mathrm{A}\left(e_{k}, v\right), \mathrm{A}\left(e_{k}, w\right)\right) \\
+\frac{4}{1+c} \sum_{k=1}^{m}\left(f^{*} \mathrm{R}_{N}\left(e_{k}, v, e_{k}, w\right)-c \mathrm{R}_{M}\left(e_{k}, v, e_{k}, w\right)\right),
\end{aligned}
$$

where

$$
\operatorname{Ric} v:=-\sum_{k=1}^{m} \mathrm{R}\left(e_{k}, v\right) e_{k}
$$

is the Ricci operator on $(M, \mathrm{~g})$ and $\left\{e_{1}, \ldots, e_{m}\right\}$ is any orthonormal frame with respect to the induced metric g.

Now let $f: M \rightarrow N$ be minimal, i. e. $\vec{H}=0$. Setting

$$
\begin{aligned}
\Psi_{c}(\vartheta)(v, w):= & -\vartheta(\operatorname{Ric} v, w)-\vartheta(\operatorname{Ric} w, v) \\
& -2 \sum_{k=1}^{m}\left(\mathrm{~s}_{M \times N}-\frac{1-c}{1+c} \mathrm{~g}_{M \times N}\right)\left(\mathrm{A}\left(e_{k}, v\right), \mathrm{A}\left(e_{k}, w\right)\right) \\
& -\frac{4}{1+c} \sum_{k=1}^{m}\left(f^{*} \mathrm{R}_{N}\left(e_{k}, v, e_{k}, w\right)-c \mathrm{R}_{M}\left(e_{k}, v, e_{k}, w\right)\right),
\end{aligned}
$$


we can write

$$
\Delta \Phi_{c}+\Psi_{c}\left(\Phi_{c}\right)=0 .
$$

To estimate the terms in $\Psi_{c}$ which contain the second fundamental tensor, we have the following statement.

Lemma 4.1. Let $\eta \in T_{p}^{\perp} M$ be a normal vector on the graph. Then for any $c \geq \lambda_{m}^{2}$ the estimate

$$
\mathrm{s}_{M \times N}(\eta, \eta) \leq \frac{c-1}{1+c} \mathrm{~g}_{M \times N}(\eta, \eta)
$$

holds. In particular,

$$
\mathrm{S}_{M \times N}(\mathrm{~A}(v, w), \mathrm{A}(v, w)) \leq \frac{c-1}{1+c}\|\mathrm{~A}(v, w)\|^{2} .
$$

Proof. Using the formulae for the singular values above, we calculate

$$
\mathrm{s}_{M \times N}\left(\xi_{i}, \xi_{j}\right)=-\delta_{i j} \leq-\frac{1-c}{1+c} \delta_{i j}, \quad 1 \leq i, j \leq n-r
$$

and

$$
\begin{aligned}
\mathrm{S}_{M \times N}\left(\xi_{i}, \xi_{j}\right) & =-\frac{1-\lambda_{i+m-n}^{2}}{1+\lambda_{i+m-n}^{2}} \delta_{i j} \\
& \leq-\frac{1-c}{1+c} \delta_{i j}, \quad n-r+1 \leq i, j \leq n .
\end{aligned}
$$

The terms involving the curvatures can be decomposed in the following way.

Lemma 4.2. Let $\left\{e_{1}, \ldots, e_{m}\right\}$ be a local g-orthonormal frame. Then for any $e_{l}$ we have

$$
\begin{aligned}
& 2 \sum_{k=1}^{m}\left(f^{*} \mathrm{R}_{N}\left(e_{k}, e_{l}, e_{k}, e_{l}\right)-c \mathrm{R}_{M}\left(e_{k}, e_{l}, e_{k}, e_{l}\right)\right) \\
& =-2 \sum_{k \neq l} f^{*} g_{N}\left(e_{k}, e_{k}\right)\left\{\left(\sigma-\sec _{N}\left(\mathrm{~d} f\left(e_{k}\right) \wedge \mathrm{d} f\left(e_{l}\right)\right)\right) f^{*} \mathrm{~g}_{N}\left(e_{l}, e_{l}\right)\right. \\
& \left.\quad+\sigma \Phi_{c}\left(e_{l}, e_{l}\right)\right\} \\
& \quad-c g_{M}\left(e_{l}, e_{l}\right) \sum_{k \neq l} \Phi_{c}\left(e_{k}, e_{k}\right)\left(\sec _{M}\left(e_{k} \wedge e_{l}\right)-\sigma\right) \\
& \quad-\frac{2 c}{1+c}\left(\operatorname{Ric}_{M}\left(e_{l}, e_{l}\right)-(m-1) \sigma \mathrm{g}_{M}\left(e_{l}, e_{l}\right)\right) \\
& \quad-\frac{2 \sigma c}{1+c}\left(\operatorname{tr}(\mathrm{s})-\mathrm{s}\left(e_{l}, e_{l}\right)\right) \\
& \quad-\frac{\sigma(1+c)}{2} \Phi_{c}\left(e_{l}, e_{l}\right)\left(\operatorname{tr}\left(\Phi_{c}\right)-\Phi_{c}\left(e_{l}, e_{l}\right)\right)
\end{aligned}
$$

Proof. We calculate

$$
\begin{aligned}
& 2 \sum_{k=1}^{m}\left(f^{*} \mathrm{R}_{N}\left(e_{k}, e_{l}, e_{k}, e_{l}\right)-c \mathrm{R}_{M}\left(e_{k}, e_{l}, e_{k}, e_{l}\right)\right) \\
& =2 \sum_{k \neq l} \sec _{N}\left(\mathrm{~d} f\left(e_{k}\right) \wedge \mathrm{d} f\left(e_{l}\right)\right) f^{*} \mathrm{~g}_{N}\left(e_{k}, e_{k}\right) f^{*} \mathrm{~g}_{N}\left(e_{l}, e_{l}\right) \\
& \quad-2 c \sum_{k \neq l} \sec _{M}\left(e_{k} \wedge e_{l}\right) \mathrm{g}_{M}\left(e_{k}, e_{k}\right) \mathrm{g}_{M}\left(e_{l}, e_{l}\right)
\end{aligned}
$$




$$
\begin{aligned}
& =-2 \sum_{k \neq l} f^{*} \mathrm{~g}_{N}\left(e_{k}, e_{k}\right)\left\{\left(\sigma-\sec _{N}\left(\mathrm{~d} f\left(e_{k}\right) \wedge \mathrm{d} f\left(e_{l}\right)\right)\right) f^{*} \mathrm{~g}_{N}\left(e_{l}, e_{l}\right)\right. \\
& \left.\quad+\sigma\left(\mathrm{g}_{M}\left(e_{l}, e_{l}\right)-f^{*} \mathrm{~g}_{N}\left(e_{l}, e_{l}\right)-\frac{1-c}{1+c}\right)\right\} \\
& +2 \sigma\left(\mathrm{g}_{M}\left(e_{l}, e_{l}\right)-\frac{1-c}{1+c}\right) \sum_{k \neq l} f^{*} \mathrm{~g}_{N}\left(e_{k}, e_{k}\right) \\
& -2 c \sum_{k \neq l}\left(\sec _{M}\left(e_{k} \wedge e_{l}\right)-\sigma\right) \mathrm{g}_{M}\left(e_{k}, e_{k}\right) \mathrm{g}_{M}\left(e_{l}, e_{l}\right) \\
& -2 c \sigma \sum_{k \neq l} \mathrm{~g}_{M}\left(e_{k}, e_{k}\right) \mathrm{g}_{M}\left(e_{l}, e_{l}\right) .
\end{aligned}
$$

Since

$$
2 \mathrm{~g}_{M}\left(e_{k}, e_{k}\right)=\mathrm{s}\left(e_{k}, e_{k}\right)+1=\left(\mathrm{s}\left(e_{k}, e_{k}\right)-\frac{1-c}{1+c}\right)+\frac{2}{1+c},
$$

the term involving the sectional curvatures of $M$ evaluates to

$$
\begin{aligned}
- & 2 c \sum_{k \neq l}\left(\sec _{M}\left(e_{k} \wedge e_{l}\right)-\sigma\right) \mathrm{g}_{M}\left(e_{k}, e_{k}\right) \mathrm{g}_{M}\left(e_{l}, e_{l}\right) \\
= & -c \mathrm{~g}_{M}\left(e_{l}, e_{l}\right) \sum_{k \neq l}\left(\mathrm{~s}\left(e_{k}, e_{k}\right)-\frac{1-c}{1+c}\right)\left(\sec _{M}\left(e_{k} \wedge e_{l}\right)-\sigma\right) \\
& -\frac{2 c}{1+c}\left(\operatorname{Ric}_{M}\left(e_{l}, e_{l}\right)-(m-1) \sigma \mathrm{g}_{M}\left(e_{l}, e_{l}\right)\right) .
\end{aligned}
$$

Further, using

$$
2 \mathrm{~g}_{M}\left(e_{k}, e_{k}\right)=1+\mathrm{s}\left(e_{k}, e_{k}\right), \quad 2 f^{*} \mathrm{~g}_{N}\left(e_{k}, e_{k}\right)=1-\mathrm{s}\left(e_{k}, e_{k}\right),
$$

for the remaining terms we get

$$
\begin{aligned}
2 \sigma\left(\mathrm{g}_{M}\left(e_{l}, e_{l}\right)-\frac{1-c}{1+c}\right) \sum_{k \neq l} f^{*} \mathrm{~g}_{N}\left(e_{k}, e_{k}\right)-2 c \sigma \sum_{k \neq l} \mathrm{~g}_{M}\left(e_{k}, e_{k}\right) \mathrm{g}_{M}\left(e_{l}, e_{l}\right) \\
=\frac{2 \sigma c}{1+c} \sum_{k \neq l}\left(f^{*} \mathrm{~g}_{N}\left(e_{k}, e_{k}\right)-\mathrm{g}_{M}\left(e_{k}, e_{k}\right)\right) \\
\quad+2 \sigma\left(\mathrm{g}_{M}\left(e_{l}, e_{l}\right)-\frac{1}{1+c}\right) \sum_{k \neq l}\left(f^{*} \mathrm{~g}_{N}\left(e_{k}, e_{k}\right)-c \mathrm{~g}_{M}\left(e_{k}, e_{k}\right)\right) \\
=-\frac{2 \sigma c}{1+c}\left(\operatorname{tr}(\mathrm{s})-\mathrm{s}\left(e_{l}, e_{l}\right)\right) \\
\quad+\sigma\left(\mathrm{s}\left(e_{l}, e_{l}\right)-\frac{1-c}{1+c}\right) \sum_{k \neq l}\left(\frac{1-c}{2}-\frac{1+c}{2} \mathrm{~s}\left(e_{k}, e_{k}\right)\right) \\
=-\frac{2 \sigma c}{1+c}\left(\operatorname{tr}(\mathrm{s})-\mathrm{s}\left(e_{l}, e_{l}\right)\right) \\
\quad+\frac{1+c}{2} \sigma \Phi_{c}\left(e_{l}, e_{l}\right)\left((m-1) \frac{1-c}{1+c}-\operatorname{tr}(\mathrm{s})+\mathrm{s}\left(e_{l}, e_{l}\right)\right) .
\end{aligned}
$$

Collecting all terms and using $\mathrm{s}=\Phi_{c}+\frac{1-c}{1+c} \mathrm{~g}$, the claim of the lemma follows.

Corollary 4.3. Let $\left\{e_{1}, \ldots, e_{m}\right\}$ be a local g-orthonormal frame. Then for any $e_{l}$ we have

$$
2 \sum_{k=1}^{m}\left(f^{*} \mathrm{R}_{N}\left(e_{k}, e_{l}, e_{k}, e_{l}\right)-c \mathrm{R}_{M}\left(e_{k}, e_{l}, e_{k}, e_{l}\right)\right)
$$




$$
\begin{aligned}
= & -2 \sum_{k \neq l} f^{*} \mathrm{~g}_{N}\left(e_{k}, e_{k}\right)\left(\sigma-\sec _{N}\left(\mathrm{~d} f\left(e_{k}\right) \wedge \mathrm{d} f\left(e_{l}\right)\right)\right) f^{*} \mathrm{~g}_{N}\left(e_{l}, e_{l}\right) \\
& -c \mathrm{~g}_{M}\left(e_{l}, e_{l}\right) \sum_{k \neq l} \Phi_{c}\left(e_{k}, e_{k}\right)\left(\sec _{M}\left(e_{k} \wedge e_{l}\right)-\sigma\right) \\
& -\frac{2 c}{1+c}\left(\operatorname{Ric}_{M}\left(e_{l}, e_{l}\right)-(m-1) \sigma \mathrm{g}_{M}\left(e_{l}, e_{l}\right)\right) \\
& -\frac{2 c \sigma}{1+c} \operatorname{tr}(\mathrm{s}) \\
& +\frac{\sigma(1-c)}{2} \Phi_{c}\left(e_{l}, e_{l}\right)\left(\operatorname{tr}\left(\Phi_{c}\right)-\Phi_{c}\left(e_{l}, e_{l}\right)\right) \\
& -\frac{2 c \sigma}{1+c}\left((m-2) \Phi_{c}\left(e_{l}, e_{l}\right)-\frac{1-c}{1+c}\right) .
\end{aligned}
$$

Proof. We note that

$$
\begin{aligned}
- & 2 \sigma \Phi_{c}\left(e_{l}, e_{l}\right) \sum_{k \neq l} f^{*} \mathrm{~g}_{N}\left(e_{k}, e_{k}\right) \\
& =-\sigma \Phi_{c}\left(e_{l}, e_{l}\right) \sum_{k \neq l}\left(1-\mathrm{s}\left(e_{k}, e_{k}\right)\right) \\
& =-\sigma \Phi_{c}\left(e_{l}, e_{l}\right)\left((m-1)-\operatorname{tr}(\mathrm{s})+\mathrm{s}\left(e_{l}, e_{l}\right)\right) \\
& =-\sigma \Phi_{c}\left(e_{l}, e_{l}\right)\left((m-1)-\operatorname{tr}\left(\Phi_{c}\right)-m \frac{1-c}{1+c}+\Phi_{c}\left(e_{l}, e_{l}\right)+\frac{1-c}{1+c}\right) \\
& =\sigma \Phi_{c}\left(e_{l}, e_{l}\right)\left(\operatorname{tr}\left(\Phi_{c}\right)-\Phi_{c}\left(e_{l}, e_{l}\right)\right)-(m-1) \frac{2 c \sigma}{1+c} \Phi_{c}\left(e_{l}, e_{l}\right) .
\end{aligned}
$$

Then from lemma 4.2 and using $\mathrm{s}=\Phi_{c}+\frac{1-c}{1+c} \mathrm{~g}$ we get

$$
\begin{aligned}
2 \sum_{k=1}^{m} & \left(f^{*} \mathrm{R}_{N}\left(e_{k}, e_{l}, e_{k}, e_{l}\right)-c \mathrm{R}_{M}\left(e_{k}, e_{l}, e_{k}, e_{l}\right)\right) \\
= & -2 \sum_{k \neq l} f^{*} \mathrm{~g}_{N}\left(e_{k}, e_{k}\right)\left(\sigma-\sec _{N}\left(\mathrm{~d} f\left(e_{k}\right) \wedge \mathrm{d} f\left(e_{l}\right)\right) f^{*} \mathrm{~g}_{N}\left(e_{l}, e_{l}\right)\right. \\
& +\sigma \Phi_{c}\left(e_{l}, e_{l}\right)\left(\operatorname{tr}\left(\Phi_{c}\right)-\Phi_{c}\left(e_{l}, e_{l}\right)\right)-(m-1) \frac{2 c \sigma}{1+c} \Phi_{c}\left(e_{l}, e_{l}\right) \\
& -c \mathrm{~g}_{M}\left(e_{l}, e_{l}\right) \sum_{k \neq l} \Phi_{c}\left(e_{k}, e_{k}\right)\left(\sec _{M}\left(e_{k} \wedge e_{l}\right)-\sigma\right) \\
& -\frac{2 c}{1+c}\left(\operatorname{Ric}_{M}\left(e_{l}, e_{l}\right)-(m-1) \sigma \mathrm{g}_{M}\left(e_{l}, e_{l}\right)\right) \\
& -\frac{2 \sigma c}{1+c} \operatorname{tr}(\mathrm{s})+\frac{2 \sigma c}{1+c}\left(\Phi_{c}\left(e_{l}, e_{l}\right)+\frac{1-c}{1+c}\right) \\
& -\frac{\sigma(1+c)}{2} \Phi_{c}\left(e_{l}, e_{l}\right)\left(\operatorname{tr}\left(\Phi_{c}\right)-\Phi_{c}\left(e_{l}, e_{l}\right)\right) .
\end{aligned}
$$

Rearranging the terms, the claim follows.

4.1. Proof of Theorem A. From the compactness of $M$ we get that the largest singular value $\lambda_{m}$ of $\mathrm{d} f$ attains its maximum at some point on $M$. Let $\lambda_{0}$ denote this maximum,

$$
\lambda_{0}^{2}:=\max _{x \in M} \lambda_{m}^{2}(x) .
$$

Then $\Phi_{\lambda_{0}^{2}}$ is non-negative definite, and at the point where $\lambda_{m}^{2}$ attains its maximum, we know that $e_{m}$ is a null-eigenvector of $\Phi_{\lambda_{0}^{2}}$. 
Lemma 4.4. Assume $\sec _{N} \leq \sigma \leq \sec _{M}$ for some $\sigma>0$ and assume $\lambda_{0}^{2}<1$. Then the map $f: M \rightarrow N$ is constant.

Proof. We first show that $\Psi_{\lambda_{0}^{2}}$ satisfies the null-eigenvector condition. Let $v$ be a null-eigenvector of the positive semi-definite tensor $\vartheta$ and extend it to a local gorthonormal frame $\left\{e_{1}, \ldots, e_{l}=v, \ldots, e_{m}\right\}$. Since $\lambda_{0}^{2}<1$, the map $f$ is strictly length-decreasing and accordingly it is $\mathrm{s}(w, w)>0$ for any vector $w \neq 0$. Also noting that $\Phi_{\lambda_{0}^{2}}(w, w) \geq 0$ for any $w$ by construction and

$$
\begin{gathered}
\operatorname{tr}(\mathrm{s})-\mathrm{s}(v, v)=\sum_{k \neq l} \underbrace{\mathrm{s}\left(e_{k}, e_{k}\right)}_{>0}>0, \\
\operatorname{tr}\left(\Phi_{\lambda_{0}^{2}}\right)-\Phi_{\lambda_{0}^{2}}(v, v)=\sum_{k \neq l} \underbrace{\Phi_{\lambda_{0}^{2}}\left(e_{k}, e_{k}\right)}_{\geq 0} \geq 0,
\end{gathered}
$$

we calculate

$$
\begin{aligned}
& \Psi_{\lambda_{0}^{2}}(\vartheta)(v, v) \stackrel{\text { Lem. }}{\geq} \underbrace{4.1}_{\geq 0} \underbrace{\frac{1-\lambda_{0}^{2}}{1+\lambda_{0}^{2}} \sum_{k=1}^{m}\left\|\mathrm{~A}\left(e_{k}, v\right)\right\|^{2}} \\
& -\frac{4}{1+\lambda_{0}^{2}} \sum_{k=1}^{m}\left(f^{*} \mathrm{R}_{N}\left(e_{k}, v, e_{k}, v\right)-\lambda_{0}^{2} \mathrm{R}_{M}\left(e_{k}, v, e_{k}, v\right)\right) \\
& \geq \frac{4 \sigma \lambda_{0}^{2}}{\left(1+\lambda_{0}^{2}\right)^{2}}(\operatorname{tr}(\mathrm{s})-\mathrm{s}(v, v)) \\
& +\sigma \Phi_{\lambda_{0}^{2}}(v, v)\left(\operatorname{tr}\left(\Phi_{\lambda_{0}^{2}}\right)-\Phi_{\lambda_{0}^{2}}(v, v)\right) \\
& \geq 0,
\end{aligned}
$$

where we have used lemma 4.2 and the curvature assumptions. Thus, the nulleigenvector condition is satisfied.

By the definition of $\lambda_{0}^{2}$, at the point $x \in M$ where the largest singular value $\lambda_{m}$ of $\mathrm{d} f$ attains its maximal value, we have $\Phi_{\lambda_{0}^{2}}\left(e_{m}, e_{m}\right)=0$, so that $\Phi_{\lambda_{0}^{2}}$ has a null-eigenvector at that point. Consequently, by the strong maximum principle (see theorem 2.2), $\Phi_{\lambda_{0}^{2}}$ has a null-eigenvector at any point of $M$.

We will now show that if $\Phi_{\lambda_{0}^{2}}$ admits a null-eigenvector at some point $x$, then $\Phi_{\lambda_{0}^{2}}$ vanishes at $x$. Since by definition $\Phi_{\lambda_{0}^{2}} \geq 0$, we can apply the second derivative test criterion at an arbitrary point $x \in M$. Using lemmas 4.1 and 4.2 , at $x$ we calculate

$$
\begin{array}{rl}
0 \stackrel{\min }{\leq} & \left(\Delta \Phi_{\lambda_{0}^{2}}\right)\left(e_{m}, e_{m}\right) \\
\stackrel{\text { Lem. }}{\leq} 4 & 4 \underbrace{\frac{\lambda_{0}^{2}-1}{1+\lambda_{0}^{2}} \sum_{k=1}^{m}\left\|\mathrm{~A}\left(e_{k}, e_{m}\right)\right\|^{2}}_{\leq 0} \\
& +\frac{4}{1+\lambda_{0}^{2}} \sum_{k=1}^{m}\left(f^{*} \mathrm{R}_{N}\left(e_{k}, e_{m}, e_{k}, e_{m}\right)-\lambda_{0}^{2} \mathrm{R}_{M}\left(e_{k}, e_{m}, e_{k}, e_{m}\right)\right) \\
\stackrel{\text { Lem. }}{\leq} \underbrace{4.2}_{\leq 0} & \underbrace{\frac{4 \sigma \lambda_{0}^{2}}{\left(1+\lambda_{0}^{2}\right)^{2}}}_{k \neq m} \sum_{k \neq m} \underbrace{\mathrm{s}\left(e_{k}, e_{k}\right)}_{>0}=0 .
\end{array}
$$


Consequently, since $\sigma>0$, we must have $\lambda_{0}^{2}=0$. Since $\lambda_{0}^{2}$ is the maximum of the largest singular value, it is $0 \leq \lambda_{1}^{2}(x)=\cdots=\lambda_{m}^{2}(x) \leq \lambda_{0}^{2}=0$.

To prove the remaining case of theorem $\mathrm{A}$, we only need to consider the case $\lambda_{0}^{2} \geq 1$.

Lemma 4.5. Assume $\sec _{N} \leq \sigma \leq \sec _{M}$ for some $\sigma>0$. Further, assume $\operatorname{tr}(\mathrm{s}) \geq 0$ and $\lambda_{0}^{2} \geq 1$. If there exists $\kappa>1$, such that $f^{*} \mathrm{~g}_{N}<\kappa^{2} \mathrm{~g}_{M}$ and

$$
\|\mathrm{A}\|^{2} \leq \frac{\kappa^{2} \sigma}{\kappa^{4}-1} \operatorname{tr}(\mathrm{s}),
$$

then $\Psi_{\lambda_{0}^{2}}$ satisfies the null-eigenvector condition.

Proof. Let $v$ be a null-eigenvector of the positive semi-definite tensor $\vartheta$ and extend it to a local g-orthonormal frame $\left\{e_{1}, \ldots, e_{l}=v, \ldots, e_{m}\right\}$. Note that $\Phi_{\lambda_{0}^{2}}(v, v) \geq 0$ by construction and consequently

$$
\operatorname{tr}\left(\Phi_{\lambda_{0}^{2}}\right)-\Phi_{\lambda_{0}^{2}}(v, v)=\sum_{k \neq l} \underbrace{\Phi_{\lambda_{0}^{2}}\left(e_{k}, e_{k}\right)}_{\geq 0} \geq 0 .
$$

From the curvature assumptions, lemma 4.1 and corollary 4.3 we obtain

$$
\begin{aligned}
\Psi_{\lambda_{0}^{2}}(\vartheta)(v, v) \stackrel{\text { Lem. }}{\geq} & -4 \frac{\lambda_{0}^{2}-1}{1+\lambda_{0}^{2}} \sum_{k=1}^{m}\left\|\mathrm{~A}\left(e_{k}, v\right)\right\|^{2} \\
& -\frac{4}{1+\lambda_{0}^{2}} \sum_{k=1}^{m}\left(f^{*} \mathrm{R}_{N}\left(e_{k}, v, e_{k}, v\right)-\lambda_{0}^{2} \mathrm{R}_{M}\left(e_{k}, v, e_{k}, v\right)\right) \\
\stackrel{\lambda_{0}^{2} \geq 1}{\text { Cor. } 4.3} & -\frac{4}{1+\lambda_{0}^{2}}\left\{\left(\lambda_{0}^{2}-1\right)\|\mathrm{A}\|^{2}-\frac{\lambda_{0}^{2}}{1+\lambda_{0}^{2}} \sigma \operatorname{tr}(\mathrm{s})\right\} .
\end{aligned}
$$

If $\lambda_{0}^{2}=1$, then

$$
\Psi_{\lambda_{0}^{2}}(\vartheta)(v, v) \geq \sigma \operatorname{tr}(\mathrm{s}) \geq 0 .
$$

so that the null-eigenvector condition is satisfied. If $\lambda_{0}^{2}>1$, also using $\lambda_{0}^{2}<\kappa^{2}$, it is

$$
\frac{\kappa^{2}}{\kappa^{4}-1}<\frac{\lambda_{0}^{2}}{\lambda_{0}^{4}-1}
$$

and accordingly

$$
\begin{aligned}
\Psi_{\lambda_{0}^{2}}(\vartheta)(v, v) & \geq-\frac{4}{1+\lambda_{0}^{2}}\left\{\left(\lambda_{0}^{2}-1\right)\|\mathrm{A}\|^{2}-\frac{\lambda_{0}^{2}}{1+\lambda_{0}^{2}} \sigma \operatorname{tr}(\mathrm{s})\right\} \\
& =-4 \frac{\lambda_{0}^{2}-1}{1+\lambda_{0}^{2}}\left\{\|\mathrm{~A}\|^{2}-\frac{\lambda_{0}^{2}}{\lambda_{0}^{4}-1} \sigma \operatorname{tr}(\mathrm{s})\right\} \\
& \geq-4 \frac{\lambda_{0}^{2}-1}{1+\lambda_{0}^{2}}\left\{\frac{\kappa^{2}}{\kappa^{4}-1}-\frac{\lambda_{0}^{2}}{\lambda_{0}^{4}-1}\right\} \sigma \operatorname{tr}(\mathrm{s}) \\
& \geq 0,
\end{aligned}
$$

so that the null-eigenvector condition is satisfied.

Let us now finish the proof of theorem A. By construction, the non-negative definite tensor $\Phi_{\lambda_{0}^{2}}$ admits a null-eigenvector at some point. By lemma 4.5, we can apply the strong maximum principle (see theorem 2.2) to conclude that $\Phi_{\lambda_{0}^{2}}$ admits a nulleigenvector everywhere on $M$, i. e. $\Phi_{\lambda_{0}^{2}}$ attains its minimal value at every point on $M$. Thus, we may apply the second derivative test criterion to $\Phi_{\lambda_{0}^{2}}$ at an arbitrary point 
$x \in M$. At $x$ consider a basis $\left\{e_{1}, \ldots, e_{m}\right\}$, orthonormal with respect to $g$ consisting of eigenvectors of $\Phi_{\lambda_{0}^{2}}$, such that $e_{m}$ is a null-eigenvector of $\Phi_{\lambda_{0}^{2}}$ and $\lambda_{m}^{2}(x)=\lambda_{0}^{2}$. From lemma 4.1 and corollary 4.3 we conclude

$$
\begin{aligned}
& 0 \stackrel{\min }{\leq}\left(\Delta \Phi_{\lambda_{0}^{2}}\right)\left(e_{m}, e_{m}\right) \\
& \leq \underbrace{\frac{4}{1+\lambda_{0}^{2}}}_{>0}(\underbrace{\left(\lambda_{0}^{2}-1\right)\|\mathrm{A}\|^{2}-\frac{\lambda_{0}^{2}}{1+\lambda_{0}^{2}} \sigma \operatorname{tr}(\mathrm{s})}_{\leq 0}) \\
& -\underbrace{\frac{4}{1+\lambda_{0}^{2}}}_{>0} \sum_{k \neq m} \underbrace{f^{*} g_{N}\left(e_{k}, e_{k}\right)}_{\geq 0}(\underbrace{\sigma-\sec _{N}\left(\mathrm{~d} f\left(e_{k}\right) \wedge \mathrm{d} f\left(e_{m}\right)\right)}_{\geq 0}) \\
& -\underbrace{\frac{4 \lambda_{0}^{2}}{\left(1+\lambda_{0}^{2}\right)^{2}}}_{\geq 0} \sum_{k \neq m} \underbrace{\Phi_{\lambda_{0}^{2}}\left(e_{m}, e_{m}\right)}_{\geq 0}(\underbrace{\sec _{M}\left(e_{k} \wedge e_{m}\right)-\sigma}_{\geq 0}) \\
& -\underbrace{\frac{4 \lambda_{0}^{2}}{\left(1+\lambda_{0}^{2}\right)^{2}}}_{\geq 0}(\underbrace{\operatorname{Ric}_{M}\left(e_{m}, e_{m}\right)-(m-1) \sigma g_{M}\left(e_{m}, e_{m}\right)}_{\geq 0}) \\
& +\underbrace{\frac{1-\lambda_{0}^{2}}{1+\lambda_{0}^{2}}}_{\leq 0} \sigma \underbrace{\Phi_{\lambda_{0}^{2}}\left(e_{m}, e_{m}\right)}_{\geq 0}(\underbrace{\operatorname{tr}\left(\Phi_{\lambda_{0}^{2}}\right)-\Phi_{\lambda_{0}^{2}}\left(e_{m}, e_{m}\right)}_{\geq 0}) \\
& -\underbrace{\frac{4 \lambda_{0}^{2}}{\left(1+\lambda_{0}^{2}\right)^{2}}}_{>0} \sigma(\underbrace{(m-2) \Phi_{\lambda_{0}^{2}}\left(e_{m}, e_{m}\right)}_{\geq 0} \underbrace{-\frac{1-\lambda_{0}^{2}}{1+\lambda_{0}^{2}}}_{\geq 0})=0 .
\end{aligned}
$$

Since all terms have the same sign, they have to vanish independently. Using $\sigma>0$ and $\lambda_{0}^{2} \geq 1$, the second line and the assumptions on $\|\mathrm{A}\|^{2}$ imply

$$
\begin{aligned}
0 & =\left(\lambda_{0}^{2}-1\right)\|\mathrm{A}\|^{2}-\frac{\lambda_{0}^{2}}{1+\lambda_{0}^{2}} \sigma \operatorname{tr}(\mathrm{s}) \\
& \leq\left(\left(\lambda_{0}^{2}-1\right) \frac{\kappa^{2}}{\kappa^{4}-1}-\frac{\lambda_{0}^{2}}{1+\lambda_{0}^{2}}\right) \sigma \operatorname{tr}(\mathrm{s}) \stackrel{\lambda_{0}^{2}<\kappa^{2}}{\leq} 0 .
\end{aligned}
$$

Since $\sigma>0$, this yields $\operatorname{tr}(\mathrm{s})=0$. From

$$
0=-\underbrace{\frac{4 \lambda_{0}^{2}}{\left(1+\lambda_{0}^{2}\right)^{2}}}_{>0} \sigma(\underbrace{(m-2) \Phi_{\lambda_{0}^{2}}\left(e_{m}, e_{m}\right)}_{\geq 0} \underbrace{-\frac{1-\lambda_{0}^{2}}{1+\lambda_{0}^{2}}}_{\geq 0})
$$

we infer $\lambda_{0}^{2}=1$. Consequently, $\operatorname{tr}(\mathrm{s})=0$ and $\lambda_{1}^{2}(x) \leq \cdots \leq \lambda_{m}^{2}(x)=\lambda_{0}^{2}=1$ force all singular values to be equal to one at any point $x \in M, \lambda_{1}^{2}(x)=\cdots=\lambda_{m}^{2}(x)=1$. Now the assumption on the second fundamental form reads

$$
\|\mathrm{A}\|^{2} \leq \frac{\kappa^{2} \sigma}{\kappa^{4}-1} \operatorname{tr}(\mathrm{s})=0,
$$

so that $\Gamma(f)$ is totally geodesic. Further, it follows that $\mathrm{g}=2 \mathrm{~g}_{M}$ and the second fundamental form also satisfies the equation

$$
0=\mathrm{A}=\mathrm{A}_{I} \oplus \mathrm{A}_{f},
$$

where $\mathrm{A}_{I}$ is the second fundamental form of the map $I:(M, \mathrm{~g}) \rightarrow\left(M, \mathrm{~g}_{M}\right)$, and $\mathrm{A}_{f}$ is the second fundamental form of the map $f:(M, \mathrm{~g}) \rightarrow\left(N, \mathrm{~g}_{N}\right)$. Consequently, 
$\mathrm{A}_{f}=0$, so that $f$ is a totally geodesic isometric immersion.

Since all singular values are the same and all vectors are null-eigenvectors of $\Phi_{\lambda_{0}^{2}}$, we can evaluate $0=\left(\Delta \Phi_{\lambda_{0}^{2}}\right)(v, v)$ for an arbitrary vector $v$. Then the third line in (4.1) yields $\sec _{N}=\sigma$ on $\mathrm{d} f(T M)$, and the fifth line implies $\operatorname{Ric}_{M}=\sigma \mathrm{g}_{M}$. In view of the curvature assumptions, the latter means $\sec _{M}=\sigma$. Thus, the claim of the theorem follows.

4.2. Proof of Corollary B. Using the assumptions on the dimensions and the notation from section 3.2, we have $r=r(x)=\operatorname{rank}(\mathrm{d} f) \leq \min \{m, n\}=n$ and $\lambda_{j}(x)=0$ for $j=1, \ldots, m-r$. Consequently,

$$
\operatorname{tr}(\mathrm{s})=\underbrace{\sum_{j=1}^{m-r} 1}_{\geq m-n}+\underbrace{\sum_{j=m-r+1}^{m} \frac{1-\lambda_{n-m+j}^{2}(x)}{1+\lambda_{n-m+j}^{2}(x)}}_{>-r}>m-n-r \geq m-2 n \geq 0,
$$

so that condition (1.1) of theorem A is fulfilled. Condition (1.2) is satisfied by assumption, so that we can draw the conclusions of theorem A. Further, since the above inequality for $\operatorname{tr}(\mathrm{s})$ is strict, the map $f: M \rightarrow N$ must be constant and the claim follows.

\section{Discussion}

We conclude by giving some remarks on the assumptions made in the theorem.

Remark 5.1. (i) Since $M$ is compact, a constant $\kappa^{2}>1$ satisfying the inequality $f^{*} \mathrm{~g}_{N}<\kappa^{2} \mathrm{~g}_{M}$ always exists.

(ii) If $\operatorname{dim} M \geq 3$, the condition on the trace (1.1) is strictly weaker than the area-decreasing condition. In view of Eq. (1.2), this is compensated by an additional requirement on the second fundamental form.

Remark 5.2. In some situations, we can exclude case (ii) of the theorem. For example, if $\operatorname{dim} M>\operatorname{dim} N$, a map $f$ satisfying the assumptions of theorem $A$ cannot be an isometric immersion, so that $f$ necessarily has to be constant.

To draw the conclusion of theorem $\mathrm{A}$, the assumptions can be weakened in various situations. Below, we will always assume the curvature conditions of theorem A to hold.

Remark 5.3 (Low dimensions). In low dimensions, one can remove the assumption on the second fundamental form.

(i) $\operatorname{dim} N=1$. In this case, any smooth minimal map $f: M \rightarrow N$ is constant $[12$, Theorem $\mathrm{C}]$.

(ii) $\operatorname{dim} M=2$. Here, the condition $\operatorname{tr}(\mathrm{s}) \geq 0$ means that $f$ is a weakly areadecreasing map. Then [12, Theorem B] implies that $f$ is either constant, or it is an isometric immersion on a non-empty, closed subset $D$ of $M$ and strictly area-decreasing on the complement of $D$.

(iii) $\operatorname{dim} M=\operatorname{dim} N=2$. Let us consider the Jacobian of the projection map $\pi_{M}: \Gamma(f) \rightarrow M$, which may be expressed as $v:=\star \Omega_{\mathrm{g}}$, where $\Omega_{\mathrm{g}}$ is the volume form on $\Gamma(f)$ induced by the metric $\mathrm{g}$ and $\star: \Omega^{k}(M) \rightarrow \Omega^{2-k}(M)$ is the Hodge star with respect to the induced metric $\mathrm{g}$. The differential equation satisfied by $v$ is essentially calculated in [15, Proposition 3.1] and is given by

$$
\Delta \ln v=-\|\mathrm{A}\|^{2}-\lambda_{1}^{2}\left\{\left(\mathrm{~A}_{11}^{1}\right)^{2}+\left(\mathrm{A}_{12}^{1}\right)^{2}\right\}-\lambda_{2}^{2}\left\{\left(\mathrm{~A}_{12}^{2}\right)^{2}+\left(\mathrm{A}_{22}^{2}\right)^{2}\right\}
$$




$$
\begin{aligned}
& -2 \lambda_{1} \lambda_{2}\left\{\mathrm{~A}_{11}^{2} \mathrm{~A}_{21}^{1}+\mathrm{A}_{12}^{2} \mathrm{~A}_{22}^{1}\right\} \\
& -\frac{1}{\left(1+\lambda_{1}^{2}\right)\left(1+\lambda_{2}^{2}\right)}\left\{\left(\lambda_{1}^{2}+\lambda_{2}^{2}\right) \sec _{M}-2 \lambda_{1}^{2} \lambda_{2}^{2} \sec _{N}\right\} .
\end{aligned}
$$

From the minimality of $\Gamma(f)$ we also get $\mathrm{A}_{11}^{2}=-\mathrm{A}_{22}^{2}$ and $\mathrm{A}_{22}^{1}=-\mathrm{A}_{11}^{1}$, so that we estimate

$$
\begin{aligned}
\Delta \ln v \leq & -\|\mathrm{A}\|^{2}-\lambda_{1}^{2}\left\{\left(\mathrm{~A}_{11}^{1}\right)^{2}+\left(\mathrm{A}_{12}^{1}\right)^{2}\right\}-\lambda_{2}^{2}\left\{\left(\mathrm{~A}_{12}^{2}\right)^{2}+\left(\mathrm{A}_{22}^{2}\right)^{2}\right\} \\
& +2\left|\lambda_{1} \lambda_{2}\right|\left\{\left|\mathrm{A}_{22}^{2} \mathrm{~A}_{21}^{1}\right|+\left|\mathrm{A}_{12}^{2} \mathrm{~A}_{11}^{1}\right|\right\} \\
& -\frac{1}{\left(1+\lambda_{1}^{2}\right)\left(1+\lambda_{2}^{2}\right)}\left\{\left(\lambda_{1}^{2}+\lambda_{2}^{2}\right) \sec _{M}-2 \lambda_{1}^{2} \lambda_{2}^{2} \sec _{N}\right\} \\
= & -\|\mathrm{A}\|^{2}-\left(\left|\lambda_{1} \mathrm{~A}_{11}^{1}\right|-\left|\lambda_{2} \mathrm{~A}_{12}^{2}\right|\right)^{2}-\left(\left|\lambda_{1} \mathrm{~A}_{21}^{1}\right|-\left|\lambda_{2} \mathrm{~A}_{22}^{2}\right|\right)^{2} \\
& -\frac{1}{\left(1+\lambda_{1}^{2}\right)\left(1+\lambda_{2}^{2}\right)}\left\{\left(\lambda_{1}^{2}+\lambda_{2}^{2}\right)\left(\sec _{M}-\sigma\right)+2 \lambda_{1}^{2} \lambda_{2}^{2}\left(\sigma-\sec _{N}\right)\right. \\
& \left.+\sigma\left(\lambda_{1}^{2}+\lambda_{2}^{2}-2 \lambda_{1}^{2} \lambda_{2}^{2}\right)\right\} .
\end{aligned}
$$

The assumption $\operatorname{tr}(\mathrm{s}) \geq 0$ is equivalent to $\lambda_{1}^{2} \lambda_{2}^{2} \leq 1$, which in turn implies the estimate

$$
\lambda_{1}^{2}+\lambda_{2}^{2}-2 \lambda_{1}^{2} \lambda_{2}^{2} \geq \lambda_{1}^{2}+\lambda_{2}^{2}-2\left|\lambda_{1} \lambda_{2}\right|=\left(\left|\lambda_{1}\right|-\left|\lambda_{2}\right|\right)^{2} \geq 0
$$

for the third curvature term. Therefore, all terms in the above differential equation are non-negative. By the maximum principle, we conclude that $\ln v$ must be constant on $M$. Evaluating $0=\Delta \ln v$, we see that $\mathrm{A}=0$ everywhere. The estimate for the curvature term yields $\lambda_{1}^{2}=\lambda_{2}^{2}$, and then $\lambda_{1}^{2}=\lambda_{2}^{2}=0$ or $\lambda_{1}^{2}=\lambda_{2}^{2}=1$ everywhere. In the latter case, the other curvature terms imply $\sec _{M}=\sigma$ and $\sec _{N}=\sigma$ on $\mathrm{d} f(T M)$.

Remark 5.4 (Weakly length-decreasing maps). By examining lemma 4.4 and the proof of theorem $A$, we note that if $\lambda_{0}^{2} \leq 1$ one does not need to impose a condition on the second fundamental form. Thus, in the case at hand, we obtain an alternative proof of $[12$, Theorem A].

Let us remark that there exists an abundance of weakly length-decreasing minimal maps which are not totally geodesic. In particular, these maps satisfy condition (1.1).

Example 5.5 (Holomorphic Maps, [12, Example 2(a)]). For complex manifolds $M$ and $N$, the graph of a holomorphic map $f: M \rightarrow N$ is automatically minimal. By a result due to Ahlfors [1] and its extension by Yau [17], for every holomorphic map $f: M \rightarrow N$ between a complete Kähler manifold $M$ with Ricci curvature bounded from below by a negative constant $-a$ and $N$ a Hermitian manifold with holomorphic bisectional curvature bounded from above by a negative constant $-b$, it is $f^{*} \mathrm{~g}_{N} \leq \frac{a}{b} \mathrm{~g}_{M}$.

\section{References}

[1] Lars V. Ahlfors, An extension of Schwarz's lemma, Trans. Amer. Math. Soc. 43 (1938), no. 3, 359-364.

[2] Klaus Ecker and Gerhard Huisken, A Bernstein result for minimal graphs of controlled growth, J. Differential Geom. 31 (1990), no. 2, 397-400.

[3] D. Fischer-Colbrie, Some rigidity theorems for minimal submanifolds of the sphere, Acta Math. 145 (1980), no. 1-2, 29-46.

[4] Richard S. Hamilton, Three-manifolds with positive Ricci curvature, J. Differential Geom. 17 (1982), no. 2, 255-306. 
[5] _ Four-manifolds with positive curvature operator, J. Differential Geom. 24 (1986), no. 2, 153-179.

[6] S. Hildebrandt, J. Jost, and K.-O. Widman, Harmonic mappings and minimal submanifolds, Invent. Math. 62 (1980/81), no. 2, 269-298.

[7] J. Jost and Y. L. Xin, Bernstein type theorems for higher codimension, Calc. Var. Partial Differential Equations 9 (1999), no. 4, 277-296.

[8] Jürgen Jost, Yuanlong Xin, and Ling Yang, The regularity of harmonic maps into spheres and applications to Bernstein problems, J. Differential Geom. 90 (2012), no. 1, 131-176.

[9] J. Jost, Y. L. Xin, and Ling Yang, The Gauss image of entire graphs of higher codimension and Bernstein type theorems, Calc. Var. Partial Differential Equations 47 (2013), no. 3-4, 711-737.

[10] Jürgen Jost, Yuan Long Xin, and Ling Yang, The geometry of Grassmannian manifolds and Bernstein-type theorems for higher codimension, Ann. Sc. Norm. Super. Pisa Cl. Sci. (5) 16 (2016), no. 1, 1-39.

[11] Guanghan Li and Isabel M. C. Salavessa, Bernstein-Heinz-Chern results in calibrated manifolds, Rev. Mat. Iberoam. 26 (2010), no. 2, 651-692.

[12] Andreas Savas-Halilaj and Knut Smoczyk, Bernstein theorems for length and area decreasing minimal maps, Calc. Var. Partial Differential Equations 50 (2014), no. 3-4, 549-577, DOI 10.1007/s00526-013-0646-0.

[13] Richard M. Schoen, The role of harmonic mappings in rigidity and deformation problems, Complex geometry (Osaka, 1990), Lecture Notes in Pure and Appl. Math., vol. 143, Dekker, New York, 1993, pp. 179-200.

[14] Knut Smoczyk, Guofang Wang, and Y. L. Xin, Bernstein type theorems with flat normal bundle, Calc. Var. Partial Differential Equations 26 (2006), no. 1, 57-67.

[15] Mu-Tao Wang, Long-time existence and convergence of graphic mean curvature flow in arbitrary codimension, Invent. Math. 148 (2002), no. 3, 525-543.

[16] - On graphic Bernstein type results in higher codimension, Trans. Amer. Math. Soc. 355 (2003), no. 1, 265-271.

[17] Shing Tung Yau, A general Schwarz lemma for Kähler manifolds, Amer. J. Math. 100 (1978), no. 1, 197-203. 